\title{
Sensitivity analysis of droplet evaporation to convection effects modelling
}

\author{
Artur Carvalho Santos*1, Fernando Luiz Sacomano Filho², Aymeric Vié ${ }^{1,3}$ \\ ${ }^{1}$ Laboratoire EM2C, CNRS, CentraleSupélec, Université Paris-Saclay, Gif-sur-Yvette, France \\ ${ }^{2}$ Laboratory of Environmental and Thermal Engineering, Universidade de São Paulo, São \\ Paulo, Brazil \\ ${ }^{3}$ Fédération de Mathématiques de CentraleSupélec, CNRS, Gif-sur-Yvette, France \\ *Corresponding author email: artur.carvalho-santos@centralesupelec.fr
}

\begin{abstract}
To predict the evaporation of droplets on the simulation of realistic turbulent spray applications, it is still today impossible to describe the whole process by discretizing each droplet and its surrounding individually. Such simulations require the use of zero-dimensional models based on the resolution of internal variables such as the droplet velocities or temperature. Among these models, we focus on the most used one: the Abramzon-Sirignano (A-S) model [1], which is implemented in several Computational Fluid Dynamics (CFD) solvers and considered as a reference. This model is also classically used for comparing different solvers on the same benchmark case, and during its implementation there are still various degrees of freedom that can be adapted to improve the fidelity or to match the constraints of the host solver: transport and thermodynamic properties, vapor pressure, film composition rules, among others. In the present work, we investigate the sensitivity of the evaporation prediction to the different submodel choices that are at the user's discretion, with a focus on droplet convection. Simulations are performed for single-component $\mathrm{N}$-dodecane droplets evaporating into air, in order to emulate the behavior of aeronautical kerosene. A parameter sweep is made for the initial conditions, in order to capture those found in a typical pre-flame region of an aeronautical combustion chamber. We investigate the overall impact of the droplet convection modelling, mainly through droplet drag laws and the Nusselt and Sherwood numbers' correlations choices.
\end{abstract}

\section{Keywords}

Evaporation, Single-Component, Droplet, Sensitivity, Spray

\section{Introduction}

Droplet evaporation is a fundamental phenomenon that must be correctly taken into account for numerical investigations performed in spray configurations. These configurations, in turn, are today very present on the aeronautical industry, since most burners inject their fuel in liquid state, creating large sums of droplets in a turbulent flow. Given the contrast between heat and mass transfer scales concerning fuel droplets and the corresponding surrounding flow, evaporation computations require modelling. Many available droplet evaporation models in the literature have been developed with spray combustion applications in mind [1] [9] [14], and the importance of correctly predicting droplet evaporation has been thoroughly emphasized for the two-phase combustion field. For instance, in [14], the author shows the progressive developments for droplet evaporation models and their subsequent impacts.

In this work, focus is given on the evaporation sub-models regarding droplet convection, through the different drag coefficients and suitable drag laws, as well as on the Nusselt and Sherwood numbers. These coefficients have been implemented mainly through semi-empirical correlations, which are often improved or revised in the literature. The main objective here is to investigate sets of parameters that are relevant for aeronautical engines, the sensitivity to these different possibilities, and the maximum deviation one can expect over the wide range of possible correlations. 
This paper is organized as follows. We first present a brief and non-exhaustive review of correlations for drag force and Nusselt and Sherwood numbers for solid particles or droplets. Then we present the selected models for our sensitivity analysis. Results are presented, focusing on the sensitivity of the results to the choice of models. The paper is then closed with some perspectives and a conclusion.

\section{Review of correlations for drag force coefficient, Nusselt and Sherwood numbers}

Originally, the first correlations for drag and heat exchange were devised for solid particles, without mass exchange, the simplest one being the analytical solution of the Stokes flow around a sphere. It leads to the Stokes drag coefficient $C_{D}=24 / R e$ and the Nusselt number $N u=2$, only valid for small particulate Reynolds numbers. Later, correlations have been proposed to account for high Reynolds number effects into both momentum and heat exchanges. Among them, one of the most widely used in spray solvers for the drag force is the Schiller and Naumann [15] one. As for the drag correlation used for the A-S model, the so-called "standard drag curve", it can be traced back to the review done by Faeth [6], where the author included a detailed discussion regarding droplet convection, in which the formulation can be linked to the works of Putnam [10]. This droplet drag formula has essentially the same structure and practical performance as those of Schiller and Naumann. For the Nusselt number, Whitaker [16] proposed a correlation that is valid up to $R e=7.6 \times 10^{4}$, which depends also on the Prandtl number, and on the ratio between the far-field and surface viscosities of the gas phase.

When it comes to droplet evaporation, additional physics can be taken into account and accordingly other models have been proposed. For instance, the drag correlations developed by Chiang, Raju and Sirignano [2] (following the initial publication of the A-S model) seem to improve the complexity of the underlying physics and are therefore of note here. The drag correlations used on the evaporation model of Miller, Harstad and Bellan [9] explicitly take into account the Stefan flow effect through a blowing velocity Reynolds number $R e_{d, b}$, and can be traced to the blowing sphere studies by Cliffe and Lever [3]. Also, since droplet evaporation models often impose modelling of mass exchanges, an additional correlation is required for the Sherwood number $S h$. Because of the analogy between heat and mass exchanges, the Sherwood correlation is generally identical to the Nusselt correlation, replacing the Prandtl number by the Schmidt number. The work of Frössling [7] yielded one the first known correlations for the Nusselt and Sherwood numbers of evaporating droplets, and the set of correlations therein is still among the most used in CFD solvers as of today. Ranz and Marshall [11] tried a different approach to correlate the $N u, S h$ numbers but in a similar imposed equation structure, yielding a quantitatively similar performance to that by Frössling. The experimental techniques, main conclusions and relevant parameter ranges of these two studies have been well documented in [8]. Later, Renksizbulut, Yuen, Nazfiger and Li [13] [12] published correlations for the $N u, S h$ numbers which explicitly take into account the effect of the Stefan flow through the mass and heat transfer Spalding numbers, $B_{M}$ and $B_{T}$, respectively.

It should be noted that in the A-S model, used as benchmark for this work, the authors opted to first evaluate the $N u, S h$ numbers through correlations that do not take into account the Stefan flow, to then apply a correction extracted from theoretical considerations for a laminar boundary layer flow past a vaporizing wedge, effectively using corrected Nusselt and Sherwood numbers labeled as $N u^{*}, S h^{*}$. The authors have cited the correlations of Frössling but have also mentioned that, in accordance with Crocco [5], for study cases where oscillatory flows are expected with velocities near zero and frequent crossing from positive to negative values and vice-versa, one should avoid these structures of correlations due to the hypersensitivity characterized by $\left.(\partial N u / \partial R e)\right|_{R e=0}=\infty$. They then presented the correlations found in the works of Clift, Grace and Weber [4] as a workaround. 


\section{Selected models for droplet vaporisation sensitivity analysis}

For this work, the A-S model was implemented using all of the original choices presented. The main hypotheses needed to derive the model include quasi-steadiness for the gaseous phase processes when compared to the liquid phase ones, and spherical symmetry for the droplet during the evaporation process. Using their film theory, integrating the species and energy conservation equations yields two possible definitions for the droplet evaporation rate:

$$
\frac{\mathrm{d} m_{d}}{\mathrm{~d} t}=\dot{m}_{d}=-2 \pi r_{d} \rho_{f} D_{f} S h^{*} \ln \left(1+B_{M}\right)=-2 \pi r_{d} \frac{\lambda_{f}}{c_{p, V}} N u^{*} \ln \left(1+B_{T}\right)
$$

where $m_{d}$ and $r_{d}$ are the mass and radius of the droplet, $c_{p, V}$ is the specific heat at constant pressure for the fuel vapor at the droplet's surface and $\rho_{f}, D_{f}, \lambda_{f}$ are the density, mass diffusivity and heat conductivity of the gas phase in the film region, evaluated using a suitable average between the fuel vapor properties at the surface and the carrier phase properties at infinity. In order to preserve the consistency between the two derivations, the mass and heat Spalding numbers $B_{M}$ and $B_{T}$ must therefore hold a relation between themselves. Since the species conservation equations are directly tied to mass exchanges, $B_{M}$ is defined and then $B_{T}$ is imposed, as follows:

$$
B_{M}=\frac{Y_{F, s}-Y_{F, \infty}}{1-Y_{F, s}}, \quad B_{T}=\left(1+B_{M}\right)^{\phi}-1, \quad \phi=\frac{S h^{*}}{N u^{*}} \frac{c_{p, V}}{c_{p, f}} \frac{1}{L e_{f}}
$$

where $Y_{F}$ is a fuel vapor mass fraction, $s$ is the subscript for surface and $L e_{f}=\lambda_{f} /\left(\rho_{f} D_{f} c_{p, f}\right)$ is the Lewis number computed for the film region. In the original A-S model, the $N u, S h$ numbers that do not take into account Stefan flow effects are corrected using the following formulation:

$$
N u^{*}, S h^{*}=2+(N u, S h-2) / F_{T, M}, \quad F_{T, M}=\frac{\left(1+B_{T, M}\right)^{0.7}}{B_{T, M}} \ln \left(1+B_{T, M}\right)
$$

And therefore, Eq. 2 is implicit, since $B_{T}$ depends on $N u^{*}$ which in turn depends on $B_{T}$, and requires an iterative procedure. The other two equations to be solved are then the droplet's temperature and velocity evolution:

$$
\begin{aligned}
\frac{\mathrm{d} T_{d}}{\mathrm{~d} t} & =\frac{\dot{m}_{d}}{m_{d} c_{p, L}}\left[\frac{c_{p, f}\left(T_{\infty}-T_{d}\right)}{B_{T}}-h_{v a p}\right] \\
\frac{\mathrm{d} U_{d}}{\mathrm{~d} t} & =\frac{\zeta}{\tau_{d}}\left(U_{\infty}-U_{d}\right)
\end{aligned}
$$

Where $U_{\infty}$ and $T_{\infty}$ are the carrier phase velocity and temperature at infinity, and $c_{p, L}$ and $h_{v a p}$ are the specific heat at constant pressure and the latent heat of vaporisation for the liquid species. For the velocity evolution, $\tau_{d}=\left(2 r_{d}^{2} \rho_{L}\right) /\left(9 \mu_{\infty}\right)$ is the relaxation time for a droplet following Stokes drag and $\zeta$ is the correction coefficient which accounts for deviations from Stokes drag. It should be noted that in this work, the liquid density and specific heat computations are temperature-dependant and thus variable. For what follows, two blocks of models are defined, one for the drag correction factor $\zeta$ laws and another for the $N u, S h$ numbers. Different models within each block were varied, one at a time, in order to evaluate the specific influence of each model. The chosen equations for each block follow below.

\section{Droplet drag factor}

The general equation to represent the droplet's velocity evolution, Equation 5, possesses a drag factor $\zeta$ which encapsulates the different choices for the drag coefficients $C_{D}$ whilst managing to preserve the classical structure of Stokes' law. In order to be consistent with this formalism, the droplet Reynolds number is defined for this block of models as being:

$$
R e_{d, \infty}=\frac{2 r_{d} \rho_{\infty}\left|U_{\infty}-U_{d}\right|}{\mu_{\infty}}
$$

The different models for the drag factor to be compared here are summarized below: 
- Standard drag curve (Reference) - subscript REF - [1], [6], [10]

$$
\zeta_{\text {REF }}=\left[1+\frac{R e_{d, \infty}^{2 / 3}}{6}\left(\frac{\mu_{\infty}}{\mu_{f}}\right)^{2 / 3}\right] \frac{\mu_{f}}{\mu_{\infty}}
$$

- Chiang, Raju and Sirignano - subscript CRS - [2]

$$
\zeta_{C R S}=\frac{1}{\left(1+B_{T}^{0.32}\right)}\left[1+0.325 R e_{d, \infty}^{0.474}\left(\frac{\mu_{\infty}}{\mu_{f}}\right)^{0.474}\right] \frac{\mu_{f}}{\mu_{\infty}}
$$

- Cliffe and Lever - subscript CL - [3]

$$
\begin{aligned}
\zeta_{C L} & =\frac{1+0.0545 R e_{d, \infty}+0.1 R e_{d, \infty}^{1 / 2}\left(1-0.03 R e_{d, \infty}\right)}{1+\alpha R e_{d, b}^{\beta}} \\
\alpha & =0.09+0.077 \exp \left(-0.4 R e_{d, \infty}\right), \quad \beta=0.4+0.77 \exp \left(-0.04 R e_{d, \infty}\right) \\
\operatorname{Re}_{d, b} & =\frac{2 r_{d} \rho_{\infty}\left|U_{\infty}-U_{b}\right|}{\mu_{\infty}}, \quad U_{b}=\frac{\left|\dot{m}_{d}\right|}{4 \pi r_{d}^{2} \rho_{\infty}}
\end{aligned}
$$

For both the REF and CRS models, film viscosity appears explicity because of the different Reynolds number definition in the original works of [1], which takes into account the film viscosity instead of the free-flow viscosity:

$$
R e_{d, f}=\frac{2 r_{d} \rho_{\infty}\left|U_{\infty}-U_{d}\right|}{\mu_{f}}
$$

\section{Nusselt and Sherwood numbers}

The Nusselt and Sherwood numbers correlations have been devised differently for each model, and the main difference apart the formulae structures chosen for the fitting is whether the correlation itself already incorporates Stefan flow effects $\left(N u^{*}, S h^{*}\right)$, or whether it is added afterwards $(N u, S h)$. We can also notice that, since the two blocks of models are varied separately, all of the Nusselt and Sherwood numbers below will include $R e_{d, f}$ as the choice of Reynolds number in order to be consistent with the formulation of the A-S evaporation model, with which all results are generated. Accordingly, the Prandtl and Schmidt numbers are defined for this film region as being $P r_{f}=\left(\mu_{f} c_{p, f}\right) / \lambda_{f}$ and $S c_{f}=\mu_{f} /\left(\rho_{f} D_{f}\right)$, respectively. The correlations for $N u$ and $S h$ investigated in the present work are the following:

- Frössling (Reference) - subscript REF - [1], [7]

$$
[N u, S h]_{R E F}=2+0.552 R e_{d, f}^{1 / 2}\left[P r_{f}, S c_{f}\right]^{1 / 3}
$$

- Clift, Grace and Weber - subscript CGW - [4]

$$
[N u, S h]_{C G W}=1+\mathcal{F}\left(1+R e_{d, f}\left[P r_{f}, S c_{f}\right]^{1 / 3}\right), \quad \mathcal{F}=\left\{\begin{array}{cc}
1 & \text { if } R e_{d, f} \leq 1 \\
R e_{d, f}^{0.077} & \text { otherwise }
\end{array}\right.
$$

- Whitaker - subscript WTK - [16]

$$
[N u, S h]_{W T K}=2+\left(0.4 R e_{d, f}^{1 / 2}+0.06 R e_{d, f}^{2 / 3}\right)\left[P r_{f}, S h_{f}\right]^{0.4}\left(\mu_{\infty} / \mu_{f}\right)^{1 / 4}
$$

- Renksizbulut, Yuen, Nazfiger and Li - subscript RYNL - [13],[12]

$$
\left[N u^{*}, S h^{*}\right]_{R Y N L}=\left(2+0.57 R e_{d, f}^{1 / 2}\left[\operatorname{Pr}_{f}, S h_{f}\right]^{1 / 3}\right)\left(1+B_{T}\right)^{-0.7}
$$

It should be noted that in the original works of Whitaker, one can only find the correlations for the Nusselt number. Due to the similarity between the models for heat and mass diffusion discussed previously, the correlation has been extended to the Sherwood number for practical purposes. 


\section{Results and discussion}

At a first moment, our implementation for the A-S model was validated using the original test case presented for a single n-decane droplet. In the following, we define a set of operating conditions that are relevant for aeronautical engines. The objective is to see the degree of sensitivity that is present on the choice of the different models, therefore providing a relative comparison between them. Considering all listed possibilities, we seek the largest deviations we can expect, and thus to see if choices inside each block of models representing convection on evaporating droplets would have a major impact or not.

Simulations are performed for single n-dodecane droplets, a surrogate for kerosene, evaporating into air for each individual model variation. The droplet initial temperature is fixed a $T_{l}(t=0)=300 \mathrm{~K}$. The fuel vapor mass fraction at infinity is set to zero, and the free-flow background pressure is $p_{\infty}=101325 \mathrm{~Pa}$. A total of 64 cases were tested for each model, corresponding to the independent change of the initial conditions presented in Table 1 . We decide to vary initial diameters, surrounding temperature, and free-flow velocity, because these are the primary parameters that will affect the droplet vaporization. In order to have a first analysis, we let aside three questions: the change in the conditions "at infinity" when a droplet meets a flame region or when a population of droplets evaporates, which would imply varying temperature and compositions, and the change in velocity due to the turbulent flow and the flow topology. This will be the subject of following studies.

Table 1. Initial conditions parameter sweep

\begin{tabular}{c|c|c}
\hline Diameter $d_{0}[\mu \mathrm{m}]$ & Surrounding temperature $T_{\infty}[K]$ & Droplet velocity $U_{d, 0}[\mathrm{~m} / \mathrm{s}]$ \\
\hline 10 & 500 & 0 \\
40 & 833.3 & 16.7 \\
70 & 1166.7 & 33.3 \\
100 & 1500 & 50 \\
\hline
\end{tabular}

Results are presented in Figs. 1-2. For each figure, the droplet lifetime $t_{\text {evap }}$ (left), the time for the droplet to reach its wet-bulb temperature $t_{T_{w b}}$ (center) and the time for the particle to reach the speed of the free-flow, $t_{U_{\infty}}$ (right) are plotted against the simulation number (ranging from 1 to 64). For both Fig. 1 and Fig. 2, the symbols represent the different initial droplet velocity for the top plot while they represent the initial droplet size for the bottom plot. The variation of surrounding temperature is depicted through the vertical lines within each image. Each color represents a different model variation. The reader must then remind that, within a figure, both trios present results from the same simulations, they are just showcased differently. This layout is chosen such that different parameters are emphasized through symbols one at a time, in order to provide clarity to the analysis.

Focusing on the physics, expected results are retrieved. The increase of the temperature at infinity decreases vaporisation and heating time scales. The momentum relaxation time scale is also subsequently decreased because of the faster diameter reduction. Concerning initial droplet velocity, we see that the closer the droplet velocity is to the gas phase one $(50 \mathrm{~m} / \mathrm{s})$, the slower the vaporization rate is. The reader can notice that when the gas and droplet velocity are equal, the momentum relaxation time is obviously zero, and no convection effects are accounted for. Finally, for the droplet diameter, we again observe an expected behaviour, as increasing initial droplet diameter increases in turn all time scales, as they are surface exchanges between the droplet and the carrier phase.

In Fig. 1, we first change the drag correlation model. The first observation is that the effect of modifying the drag correlation is clearly more visible in the momentum relaxation time, which is not surprising. For the evaporation and heating times, the sensitivity is less visible. The model that is departing the most from the baseline A-S one is CRS, and the largest sensitivity is found 
for large droplets, the initial velocity and temperature having a weak influence on the deviations between models.

In Fig. 2, we now change the Nusselt and Sherwood correlations. Therein, we can see equivalent sensitivity for all time scales. A first observation is that RYNL behaves quite differently from the three other models: the droplet lifetimes predicted by this model tend to be always higher and roughly by the same amount, which translates to an increasingly higher percentage difference as the free-flow temperature rises. This may be due to the fact that this model does not have the guarantee for its $N u^{*}, S h^{*}$ numbers to converge to 2 when there is no convection, since the factor $\left(1+B_{T}\right)^{-0.7}$ encapsulates everything in Eqs. 13. Apart for this strong deviation of $R Y N L$, the other models seems to behave quite similarly, with little visible deviations. All of the time scales are higher using models WTK and CGM compared to the reference model REF. Also, it is seen that no appreciable difference in the metrics is captured whenever there is no relative velocity from the droplet to the gas $\left(U_{d, 0}=50 \mathrm{~m} / \mathrm{s}\right)$, for all models except for the $R Y N L$ one in $N u, S h$ (squares on the upper trio). Indeed, a single droplet simulation was realized for a case where no convection effects are expected and all models yielded constant $N u^{*}=2$ numbers whereas $R Y N L$ yielded a varying $1.2<N u^{*}<1.43$. Again, bigger droplets tend to showcase more differences between models, because a larger droplet lifetime is expected.
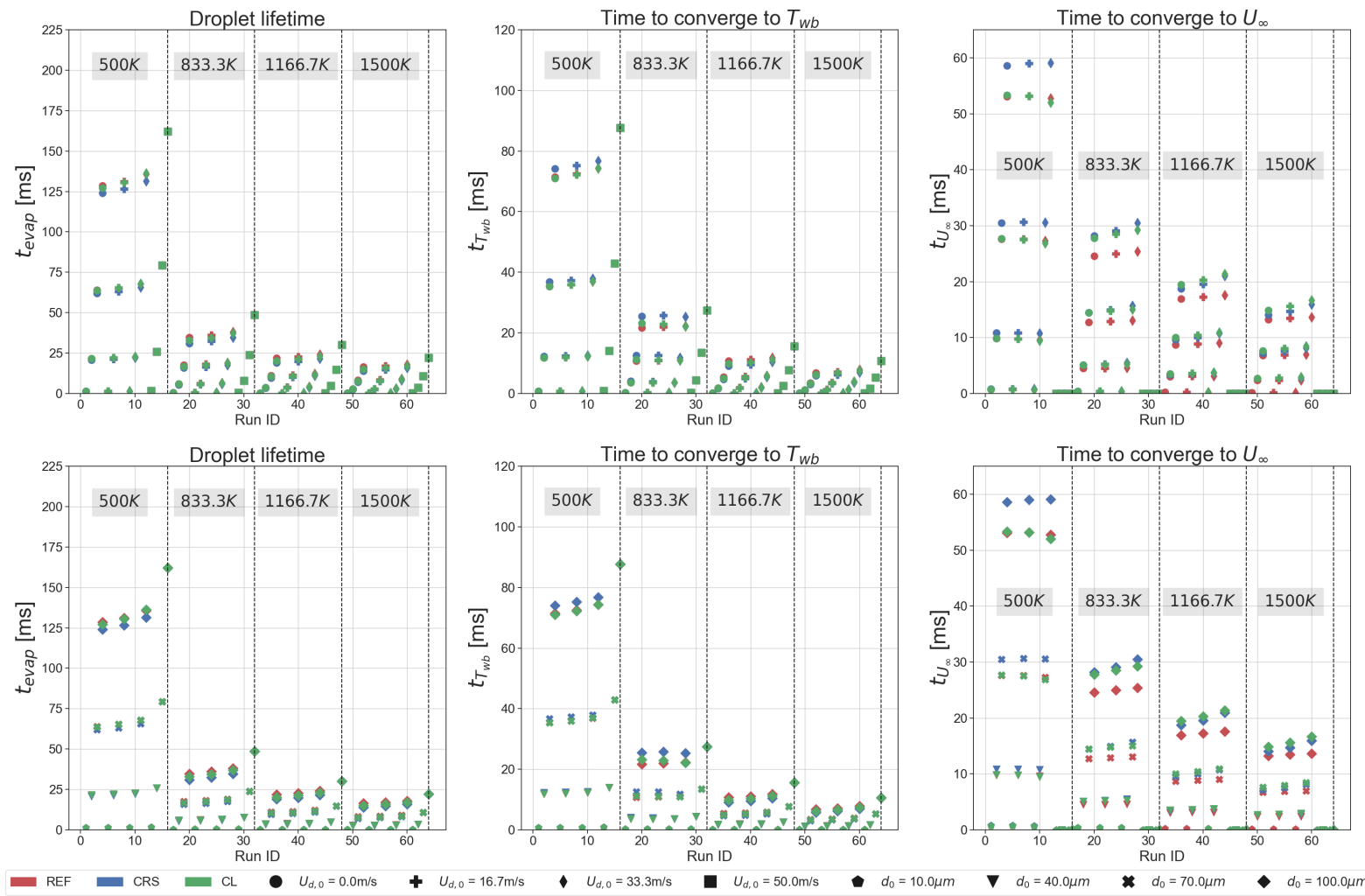

Figure 1. Droplet evaporation time scales for operating conditions in Table 1, varying the drag model: evaporation (left), heating(center), and drag relaxation (right) times. The abscissa corresponds to the simulation number. Vertical lines separate simulations with the same temperature at infinity. In the top plots, different symbols are used for each initial droplet velocity. In the bottom plots, different symbols are used for each initial droplet diameter.

To summarize these results, we define a metric that measures the maximum normalized deviation that we expect for a set of modelling choices, captured over all 64 simulations. This is done for each block individually (subscripts $\zeta$ and $N u, S h$ ) as well as for both blocks combined (subscript $g l o b a l$ ), in order to try to essentially represent the maximum impact one can expect for each metric when varying only each model independently. This metric is defined below.

$$
\Delta_{\text {max }}^{*}=\max _{k=1, N_{\text {cases }}}\left(\frac{\max _{l=1, N_{\text {models }}}\left(\tau_{k, l}\right)-\min _{l=1, N_{\text {models }}}\left(\tau_{k, l}\right)}{\operatorname{mean}_{l=1, N_{\text {models }}}\left(\tau_{k, l}\right)}\right)
$$

where $\tau$ is replaced by the appropriate time scale. Since the RYNL model proved to be quite 

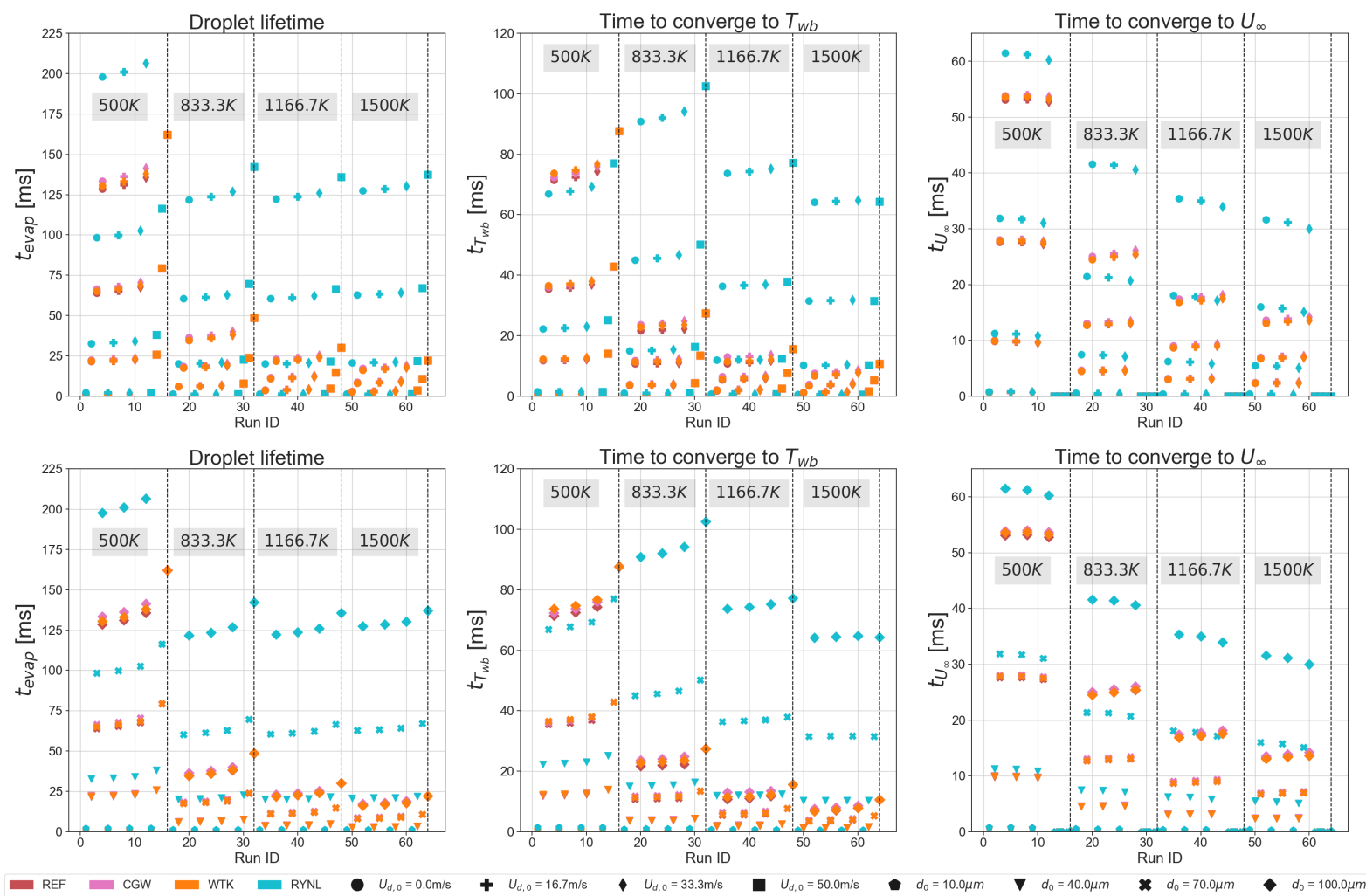

Figure 2. Droplet evaporation time scales for operating conditions in Table 1, varying the Nusselt and Sherwood correlations: evaporation (left), heating(center), and drag relaxation (right) times. The abscissa corresponds to the simulation number. Vertical lines separate simulations with the same temperature at infinity. In the top plots, different symbols are used for each initial droplet velocity. In the bottom plots, different symbols are used for each initial droplet diameter.

discrepant from the other ones, it has been omitted from these final results since otherwise it would bias the comparisons between all the other models. Results are summarized in Table 2.

Table 2. Maximum of largest relative differences normalized by average value for each metric

\begin{tabular}{c|c|c|c}
\hline Metric & $\Delta_{\text {max }, \zeta}^{*}$ & $\Delta_{\text {max }, \text { NuSh }}^{*}$ & $\Delta_{\text {max }, \text { global }}^{*}$ \\
\hline$t_{\text {evap }}$ & $16.0 \%$ & $5.9 \%$ & $20.3 \%$ \\
$t_{T_{w b}}$ & $17.5 \%$ & $12.6 \%$ & $36.9 \%$ \\
$t_{U_{\infty}}$ & $19.6 \%$ & $4.5 \%$ & $20.5 \%$ \\
\hline
\end{tabular}

As seen in Table 2, it is clear that in general the models for the drag factors impact more these key metrics than the choice of the $N u, S h$ correlations (when excluding the $R Y N L$ model). It is also seen that the global results tend to exacerbate these differences - this essentially would mean that the most extreme cases for drag models and for $N u, S h$ models when compared tend to be even more different, which is not an obvious conclusion - It should be reiterated that the average in the denominator of Equation 14 is being computed again for the global case, therefore no "a priori" conclusion is possible. The difference is particularly strong for the $t_{T_{w b}}$ metric, where a maximum of $36.9 \%$ has been obtained. This is not an aberrant value, since values over $30 \%$ have also appeared multiple times for this metric in our study. Of particular remark is the fact that all of the spikes between models for this $t_{T_{w b}}$ metric occur for a free-flow temperature of $T_{\infty}=1166.7 \mathrm{~K}$, and also generally for bigger droplets. 


\section{Conclusions}

In this work, several correlations for momentum, heat and mass exchanges of evaporating droplets have been tested using as a base model the one of Abramzon and Sirignano [1]. This benchmark did not aim at validating one model or another, but instead focused at highlighting the sensitivity to these choices of correlations. The chosen correlations are based on the ones used more often in the literature, and this choice is not expected to be fully exhaustive. Results have shown an overall sensitivity of about $20 \%$ on the vaporization time and velocity relaxation time, and about $37 \%$ on the thermal relaxation time. In accordance to such large sensitivity, particular attention should be given regarding the validation data and test cases of these models, such that even if models perform differently, the choice of a model could be made based on the similarity between the original experiment/correlation conditions and the actual application case of the user. For this matter, the reviews done by Faeth [6] and Fuchs [8] are quite exhaustive and are therefore recommended. However, more studies are called upon in order to develop and validate more accurate correlations, as reasons to choose one correlation over another among existing ones may not always be strong or clear enough, as models can be quite generic in scope and overlaps for their application may occur.

\section{Acknowledgements}

The support of the ANR Project MIMETYC (ANR-17-CE22-0003) is acknowledged.

\section{References}

[1] Abramzon, B., Sirignano, W. A., 1989, Int. J. Heat Mass Transfer, 32 (9), pp. 1605-1618.

[2] Chiang, C. H., Raju, M. S., Sirignano, W. A., 1992, Int. J. Heat Mass Transfer, 35 (5), pp. 1307-1324

[3] Cliffe, K. A., Lever, D. A., 1985, Int. J. Num. Methods Fluids, 5, pp. 709-725

[4] Clift, R., Grace, J.R., Weber, M. E., 1978, "Bubbles, Drops and Particles". Academic Press.

[5] Crocco, L., 1965, Tenth Symposium (International) on Combustion, pp. 1101-1128

[6] Faeth, G. M., 1977, Prog. Energy Combust. Sci., 3, pp. 191-224

[7] Frössling, N., 1938, Gerlands Beitr. Geophys., 52, pp. 170-216

[8] Fuchs, N. A., 1959, "Evaporation and Droplet Growth in Gaseous Media".

[9] Miller, R. S., Harstad, K., Bellan, J., 1998, Int J. Multiphase Flow, 24, pp. 1025-1055

[10] Putnam, A., 1961, ARS Journal, 31, pp. 1467-1468

[11] Ranz, W. E., Marshall Jr., W. R., 1952, Chem. Eng. Prog., 48

[12] Renksizbulut, M., Nazfiger, R., Li, X., 1991, Chem. Eng. Sci., 46 (9), pp. 2351-2358

[13] Renksizbulut, M., Yuen, M.C., 1983, Transact. ASME, 105, pp. 384-388

[14] Sazhin, S., 2014, "Droplets and Sprays". Springer.

[15] Schiller, L., Naumann, Z., 1935, VDI Zeitung, 77, pp. 318-320

[16] Whitaker, S., 1972, AIChE Journal, 18 (2), pp. 361-371 